\title{
EQUILIBRIO DE BIOSORCIÓN DE PLOMO (II) Y \\ CARACTERIZACIÓN MEDIANTE FT-IR Y SEM-EDAX EN ALGA Ascophyllum nodosum
}

\author{
Neptalí Ale Borja, ${ }^{a}$ Víctor García Villegas, ${ }^{a}$ Andrea Yipmantin Ojeda, ${ }^{\mathrm{b}}$ Enrique Guzmán \\ Lezama, ${ }^{\mathrm{a}, \mathrm{b}}$ Holger Maldonado García. ${ }^{\mathrm{a}, \mathrm{b}}$
}

\begin{abstract}
RESUMEN
Se ha estudiado la biosorción de plomo en el alga Ascophyllum nodosum a $20^{\circ} \mathrm{C}$ y un $\mathrm{pH}$ inicial 4-5 empleando los modelos de isoterma de Langmuir y Freundlich. Para la estabilización del alga se empleó $\mathrm{CaCl}_{2}$. La granulometría empleada en el biosorbente fue 0-75 $\mu \mathrm{m}$. En el análisis por espectroscopía infrarrojo con transformada de Fourier, FTIR, se observó una pequeña disminución en la señal en torno al grupo carbonilo para la muestra reticulada antes de la biosorción con el metal y después $\left(1600,92 \mathrm{~cm}^{-1}\right.$ a 1573,91 $\mathrm{cm}^{-1}$ ) y a $1400 \mathrm{~cm}^{-1}$ (que pasó de $1415,75 \mathrm{~cm}^{-1}$ a $1409,96 \mathrm{~cm}^{-1}$ ), sugiriendo un mecanismo de biosorción del metal por quelación bidentada con los grupos carboxilos pertenecientes a las unidades monoméricas de alginato. La banda ubicadas en $3305.99 \mathrm{~cm}^{-1}$ permitió reconocer a los grupos hidroxilos presentes en el biosorbente. La microscopía electrónica de barrido, SEM, comprobó la heterogeneidad de las partículas de biosorbente. En el espectro de rayos $\mathrm{X}$ con energía dispersiva, EDAX, se observó una señal muy intensa para el carbono y otra de menor intensidad para el oxígeno, características en el biopolímero. La señal del calcio pudo ser reconocida en el espectro EDAX de la muestra después del pretratamiento. Los dos picos del espectro correspondientes al elemento plomo se observaron en la muestra después del proceso de biosorción. Los datos experimentales para el equilibrio de biosorción presentaron mayor coeficiente de correlación para el modelo de isoterma de Langmuir, alcanzando una capacidad de sorción máxima, $q_{\max }$, igual $228 \mathrm{mg} / \mathrm{g}$. La concentración residual de plomo (II), $C_{e q}$, en las soluciones después de haber alcanzado el equilibrio, se determinó a las $72 \mathrm{~h}$ de iniciado el proceso, mediante espectroscopía de absorción atómica. Para el procesamiento de datos y tratamiento estadístico se empleó el programa informático Origin 6.0.
\end{abstract}

Palabras clave: Biosorción, Ascophyllum nodosum, plomo, isoterma, Langmuir, Freundlich

\section{EQUILIBRIUM OF LEAD (II) BIOSORPTION AND CHARACTERIZATION THROUGH FT-IR AND SEM-EDAX ON Ascophyllum nodosum SEAWEED}

\footnotetext{
ABSTRACT

Lead (II) biosorption on Ascophyllum nodosum seaweed at $20^{\circ} \mathrm{C}$ and initial $\mathrm{pH} 4-5$ was studied, by using models of Langmuir and Freundlich isotherms. In order to establish seaweed, $\mathrm{CaCl}_{2}$ was used. The granulometry used in the biosorbent was 0-75 $\mu \mathrm{m}$. In Fourier

a Facultad de Química e Ing. Quimica., Universidad Nacional Mayor de San Marcos, Lima 1 nalebunmsm@gmail.com

b Facultad de Ciencias y Filosofía Alberto Cazorla Talleri. Departamento de Ciencias Exactas-Sección Química, Universidad Peruana Cayetano Heredia, Lima 31
} 
transform infrared spectroscopy, FT-IR, a shift in the signal of carbonyl group of crosslinked sample before metal biosorption and after $\left(1600.92 \mathrm{~cm}^{-1}\right.$ to $\left.1573.91 \mathrm{~cm}^{-1}\right)$ and $1400 \mathrm{~cm}^{-1}$ (that was from $1415.75 \mathrm{~cm}^{-1}$ to $1409.96 \mathrm{~cm}^{-1}$ ) was observed, suggesting a chelating (bidentate) character of the metal biosorption onto carboxylic groups that belongs to the units of alginate monomeric. The bands at $3305.99 \mathrm{~cm}^{-1}$ allowed the recognition of hydroxyl groups in the biosorbent. The scanning electronic microscopy, SEM, verified heterogeneity of biosorbent particles. In the energy-dispersive X-ray spectroscopy, EDAX, a very strong signal in the carbon and another less strong for the oxygen characteristic of the biopolymer were observed.

The signal of calcium could be recognized in EDAX spectra of the sample after pre-treated. The two peaks that correspond to lead element were observed in the sample after biosorption process. Experimental data of biosorption equilibrium had greater correlation coefficient to Langmuir isotherm model, reaching the maximum sorption capacity, $q_{\max }$, of $228 \mathrm{mg} / \mathrm{g}$. Lead (II) residual concentration, $C_{e q}$, in the solutions after getting equilibrium, was determined after $72 \mathrm{~h}$ starting the process by atomic absorption spectroscopy. For data processing and statistic treatment, Origin 6.0 informatics program was used.

Key words: Biosorption, Ascophyllum nodosum, lead, isotherm, Langmuir, Freundlich.

\section{INTRODUCCIÓN}

Desde hace varias décadas se estudia y desarrolla métodos para solucionar los problemas derivados de la contaminación que producen los metales tóxicos provenientes de la actividad minera, metalúrgica e industrial. Se dispone de métodos de precipitación, membranas semipermeables, resinas de intercambio iónico y ósmosis reversa. Si bien cada técnica es efectiva para una situación en particular, estos procesos presentan grandes dificultades al ser aplicados y, por ello, son poco convenientes desde el punto de vista económico. Esto ha orientado la búsqueda de métodos más económicos y de fácil aplicación hacia otro tipo de procesos. En la década pasada, los procesos biotecnológicos han llamado la atención de la comunidad científica por la variedad de métodos detoxificantes de metales pesados. Dentro de este contexto, la biotecnología ha separado dos grandes áreas dentro del mismo objetivo: bioacumulación y biosorción. Se entiende por bioacumulación al proceso activo de eliminación de contaminantes mediante mecanismos metabólicos involucrando biomasa viviente, y biosorción como un proceso pasivo mediante un mecanismo propiamente fisicoquímico, por medio de biomasa inerte. Por ende, la biosorción es un área de investigación con muchos aportes a la comunidad industrial, velando por su integridad, brindándole alternativas tecnológicas y económicamente viables; además, es considerada una tecnología "limpia" para la eliminación de contaminantes ambientales presentes en aguas residuales o de desecho de actividades productivas. ${ }^{1-4}$ La biomasa seca de organismos animales y vegetales o residuos de cultivos de fermentación, algas que en su estructura química poseen polímeros como alginatos, celulosa, lignina, carragenano, mureína, quitina, quitosano, entre otros, los cuales presentan grupos funcionales: tales como aminas, amidas, carboxilatos, hidroxilos, fosfatos, etc; que les confieren la capacidad de fijar o retener especies metálicas.

\section{Equilibrio de biosorción}

El modelo de Freundlich, que es el modelo más sencillo, es un modelo de tipo monocapa; pero 
las energías de sorción no son las mismas en todos los puntos de la superficie del adsorbente y algunas interacciones pueden existir entre las moléculas sorbidas. En relación a la ecuación de Freundlich, el coeficiente $n$ es mayor que 1, la isoterma es favorable. Cuanto más alto sea el coeficiente $\mathrm{n}$, más favorable es la isoterma. ${ }^{5}$ Los parámetros $k \mathrm{y} n$, serán determinados al ajustar los valores experimentalmente obtenidos.

$$
q=k C_{e q}^{1 / n}
$$

Donde k y n son las constantes de Freundlich. Esta ecuación puede ser fácilmente linealizada graficando log q vs log Ce en la ecuación:

$$
\ln q=\ln k+\frac{1}{n}\left(\ln C_{e q}\right)
$$

Las constantes $\mathrm{k}$ y $1 / \mathrm{n}$ corresponden al intercepto y la pendiente, respectivamente.

La ecuación de Langmuir fue establecida sobre consideraciones cinéticas para describir la adsorción de gases sobre sólidos. Esta ecuación fue derivada para los sistemas líquido/sólido. El modelo se funda sobre tres hipótesis: la sorción ocurre sobre una sola capa del adsorbente; las energías de sorción son distribuidas de manera homogénea sobre toda la superficie del adsorbente y no existen interacciones laterales entre las moléculas adsorbidas. ${ }^{5}$

$$
q=\frac{q_{m a s} b C_{e q}}{1+b C_{e q}}
$$

Donde:

$q_{\max }=$ es la máxima capacidad de sorción bajo las condiciones dadas.

$b=$ es el coeficiente relativo de afinidad entre el sorbato y el sorbente.

Puede ser linealizada graficando $1 / \mathrm{q}$ vs $1 / C_{e q}$ en la ecuación:

$$
\frac{1}{q}=\frac{1}{b q_{\max }}+\frac{1}{b q_{\max }}\left(1 / C_{e q}\right)
$$

o $\left(C_{e q} / \mathrm{q}\right)$ vs $C_{e q}$ en la ecuación:

$$
\frac{C_{e q}}{q}=\frac{1}{b q_{\max }}+\frac{1}{q_{\max }}\left(C_{e q}\right)
$$


El modelo de Langmuir, frecuentemente usado, contiene dos parámetros $\left(q_{\max } \mathrm{y} b\right)$ que son fácilmente entendibles a partir de que reflejan dos importantes características del proceso de sorción:

- La máxima capacidad de sorción, es decir, la máxima cantidad en mg del metal que se podrá retener por cada gramo de sorbente.

- El volumen en $\mathrm{mL}$ de solución por peso en mg de adsorbente. Los parámetros $q_{\max } \mathrm{y}$ b se determinarán al ajustar los datos de experimentación obtenidos.

El modelo de Langmuir considera una capa monomolecular por deposición de iones metálicos que implica un acomodamiento. En los procesos de biosorción no se puede afirmar que ocurra exactamente así y no se puede generalizar el mecanismo involucrado. El concepto usual del sorbente de fase sólida con poros físicos en su área de superficie puede no estar tan cerca de la estructura real, apariencia y comportamiento de los materiales de biosorción ${ }^{5,6}$.

Actualmente se sabe que, como ocurre con el proceso de intercambio iónico, éstos cumplen aparentemente un papel muy importante en la bioadsorción; al menos un ion de la estructura molecular del sorbente es intercambiado por otro del mismo tipo de carga que ingresa. Una característica importante de la isoterma de sorción es una región inicial creciente que significa que existe gran afinidad por las especies y es indicada por el coeficiente b de la expresión de Langmuir. A menor valor de b existe mayor afinidad. Para determinar la eficiencia hay que observar el qmax y la pendiente del proceso inicial de sorción que es expresada por el parámetro b de la expresión de Langmuir.,

En este artículo se estudió el equilibrio de biosorición de $\mathrm{Pb}^{2+}$ en el alga Ascophyllum nodosum mediante los modelos de Langmuir y Freundlich. Los análisis FT-IR, SEM - EDAX y $\mathrm{pKa}$ permitieron el reconocimiento de los principales grupos funcionales característicos, la morfología y composición.

\section{Obtención del alga}

\section{PARTE EXPERIMENTAL}

El Ascophyllum nodosum fue proporcionado por el Dr. Eric Guibal del laboratorio de investigación de Ing. Industrial y Medio Ambiente de la Escuela de Minas de Alés-Francia.

\section{Pretratamiento del alga}

El alga fue lavada con agua desionizada y secada en una estufa de vacío a $45^{\circ} \mathrm{C}$. Luego se molió y tamizó para obtener diferentes tamaños de partículas $(\mathrm{G}<5 \mu \mathrm{m}$ y $75 \mu \mathrm{m}<\mathrm{G}<180 \mu \mathrm{m})$; el tamizado se suspendió en una solución de $\mathrm{HCl} 0,1 \mathrm{~N}$ y se puso en agitación magnética constante (LAB-LINE) por una hora con el objeto de protonar los sitios activos. Se filtró al vacío y lavó con abundante agua desionizada para eliminar el exceso de ácido. Se dejó secar durante $24 \mathrm{~h}$ a temperatura ambiente. Finalmente, se refrigeró en frascos herméticos hasta su uso.

\section{Estabilización de Ascophyllum nodosum}


Es un proceso que consiste en hacer reaccionar químicamente el material de desecho biológico o biopolímero para mantener su estructura y estabilidad y hacerlos resistentes a $\mathrm{pH}$ ácidos y así se evita la lixiviación de la materia orgánica que consiste en que un disolvente líquido que pasa a través de un sólido pulverizado produce la elución de uno o más componentes del sólido. Este fenómeno de disolución implica la formación de un ion complejo; en el caso del Ascophyllum nodosum el alginato se solubiliza en el medio debido a que el agua es un agente de lixiviación que afecta la estructura y estabilidad del biopolímero y, además, afecta las propiedades de adsorción de metales.

Este proceso se realizó mezclando $20 \mathrm{~g}$ de biomasa seca con $100 \mathrm{~mL}$ de solución de $\mathrm{CaCl}_{2}$ 0,2 $\mathrm{M}$ a pH 5 ajustándolo con $\mathrm{NaOH} 1 \mathrm{M}$ y $\mathrm{HCl} 1 \mathrm{M}$ y en agitación durante $24 \mathrm{~h}$. Al término del tratamiento la biomasa fue lavada con abundante agua purificada por medio de un sistema de ósmosis reversa para remover cualquier exceso de $\mathrm{CaCl}_{2}$. El filtrado fue descartado y el alga reticulada fue secada a $45^{\circ} \mathrm{C}$ en una estufa al vacío durante $24 \mathrm{~h}$.

Durante el proceso de reticulación se producen dos fenómenos químicos importantes: Primero se produce la protonación de la biomasa con $\mathrm{HCl}$, de esta forma el protón desplaza a los iones de los metales: $\mathrm{K}^{+}, \mathrm{Na}^{+}, \mathrm{Ca}^{2+} \mathrm{y} \mathrm{Mg}^{2+}$ adsorbidos por los grupos funcionales (ácido carboxílico, ácido sulfónico y otros) del alga. En segundo lugar, se hace reaccionar la biomasa con una solución acuosa de un ión dado en alta concentración $\left(\mathrm{Ca}^{2+}\right)$ para que este ocupe la mayoría de grupos funcionales cercanos. ${ }^{7}$

\section{Determinación del pKa}

Se agregó $0,259 \mathrm{~g}$ de alga $(0-75 \mu \mathrm{m})$ pretratada con $\mathrm{HCl}$ al $100 \mathrm{~mL}$ de solución de $\mathrm{KCl}$ $0,1 \mathrm{~N}$ con el fin de mantener una fuerza iónica estable durante toda la titulación. Se tituló con una solución valorada de $\mathrm{KOH} 0,2446 \mathrm{~N}$ en el intervalo de $\mathrm{pH} 2$ hasta 12. La titulación se realizó a $25^{\circ} \mathrm{C}$, manteniendo el recipiente con burbujeo constante de nitrógeno para evitar la presencia de oxígeno y dióxido de carbono.

El pKa fue obtenido a partir del pH correspondiente al momento en el cual la volumen de solución de $\mathrm{NaOH}$ añadido es igual a la mitad del volumen valorante $\left(\mathrm{V}_{\mathrm{A}}\right)$. En esta condición se cumple que el número de equivalentes de los ácidos $1,4 \beta-\mathrm{D}$ manurónico $(\mathrm{M})$ y $\alpha$-Lgulurónico $(\mathrm{G})$ en sus formas protonadas es igual al número de equivalentes de los mismos en forma aniónica.

\section{Isoterma de adsorción}

La muestra seca y pulverizada fue clasificada de acuerdo a tamaño de partícula mediante el método de molienda y tamizado. Asimismo, se comprobó que a $\mathrm{pH} 4$ se produce la máxima capacidad de sorción. Para los estudios de equilibrio se preparó soluciones de concentraciones variables en un rango de 20 a 100 ppm de $\mathrm{Pb}\left(\mathrm{NO}_{3}\right)_{2}$ y 15 a $25 \mathrm{mg}$ de biosorbente $(0-75 \mu \mathrm{m})$ $/ 150 \mathrm{~mL}$ al $\mathrm{pH}$ óptimo determinado anteriormente ( $\mathrm{pH} 4)$. Se colocó en agitación a $250 \mathrm{rpm}$ por 72 horas a temperatura ambiental, luego se filtró y determinó la concentración del metal por la técnica de Absorción Atómica. 


\section{Caracterización}

Las muestras sin estabilizar, después de su estabilización y después de la biosorción del metal fueron caracterizadas mediante el método de FT-IR y SEM-EDAX.

\section{Análisis por FT-IR}

\section{RESULTADOS Y DISCUSIÓN}

Se realizó el análisis por Espectroscopía de Infrarrojo con transformada de Fourier (FT-IR) para la caracterización de grupos funcionales del bioadsorbente antes de la estabilización con cloruro de calcio, después de la estabilización y después de la biosorción de plomo. En general, los cambios producidos en las bandas de un espectro IR para una muestra luego de un proceso determinado están relacionados con los cambios en la composición de los grupos funcionales de la estructura de dicha muestra. La figura 1 representa los espectros obtenidos del alga Ascophyllum nodosum antes y después del proceso de biosorción de $\mathrm{Pb}$ (II).

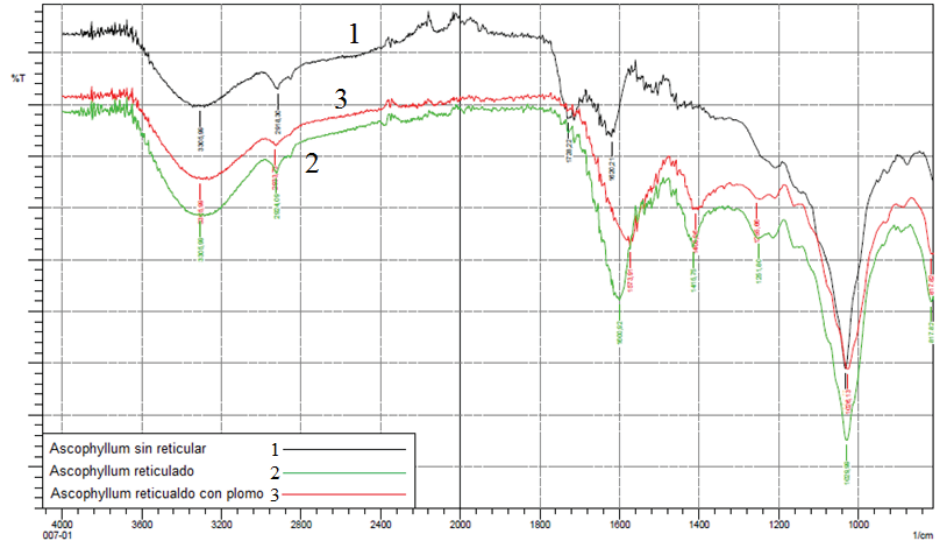

Figura 1. Espectro FT-IR del Ascophyllum nodosum

Las bandas ubicadas en 3305,99 $\mathrm{cm}^{-1}$ corresponden a los grupos hidroxilo. Después de la biosorción de $\mathrm{Pb}$ (II), los espectros FT-IR han cambiado poco. La tabla 1 muestra las bandas de estiramiento asimétrico de los grupos carboxilato, (VCOO) $)_{\text {asim }}$, alrededor de $1620,21 \mathrm{~cm}^{-1}$ para la muestra sin reticular, $1600 \mathrm{~cm}^{-1}$ para la muestra reticulada y $1563,91 \mathrm{~cm}^{-1}$ para la muestra reticulada después de la biosorción de $\mathrm{Pb}$ (II) y de estiramiento simétrico de los grupos carboxilato, (VCOO) $)_{\text {sim }}$, de $1415,75 \mathrm{~cm}^{-1}$ para la muestra reticulada y $1409,96 \mathrm{~cm}^{-1}$ para la muestra reticulada después de la biosorción con el metal. Las pequeñas disminuciones que se observan en torno a $1600 \mathrm{~cm}^{-1}$ para la muestra reticulada antes de la biosorción y después $\left(1600,92 \mathrm{~cm}^{-1}\right.$ a $1573,91 \mathrm{~cm}^{-1}$ ) y a $1400 \mathrm{~cm}^{-1}$ (que pasó de 1415,75 $\mathrm{cm}^{-1}$ a 1409,96 $\mathrm{cm}^{-1}$ ) también han sido observadas por Sheng et al. ${ }^{8}$ Esto se debe a que la densidad de carga, el radio y el peso atómico del catión cambian, creando un nuevo ambiente en todo el grupo carbonílico. Por lo tanto se justifica un cambio en la banda. Estos cambios en los valores sugieren un mecanismo de biosorción del metal por quelación bidentada en los grupos carboxilo. ${ }^{8}$ La probable estructura de la unión metálica en un alga marrón tiene la forma: ${ }^{8}$ 


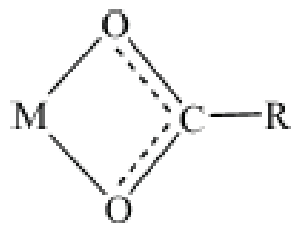

Figura 2. Representación del mecanismo de quelación bidentada. ${ }^{8}$

Las bandas en torno a $2920 \mathrm{~cm}^{-1}$ y que se superponen a la banda ancha perteneciente al grupo hidroxilo $\left(3305,99 \mathrm{~cm}^{-1}\right)$ corresponden a la absorción del enlace C-H alifático $\left(2918,30 \mathrm{~cm}^{-1}\right.$ para la muestra antes de reticular, $2924.09 \mathrm{~cm}^{-1}$ para la muestra reticulada y $2933,77 \mathrm{~cm}^{-1}$ para la muestra reticulada después de la biosorción con el metal) y finalmente las bandas que aparecen en torno a $1030 \mathrm{~cm}^{-1}\left(1031,92 \mathrm{~cm}^{-1}\right.$ para la muestra antes de reticular, $1029,99 \mathrm{~cm}^{-1}$ para la muestra reticulada y $1026,13 \mathrm{~cm}^{-1}$ para la muestra reticulada después de la biosorción con el metal) corresponden a la absorción de tensión de enlace C-O.

Tabla 1. Principales señales del espectro FT-IR para Ascophyllum nodosum.

\begin{tabular}{lccc}
\hline & \multicolumn{3}{c}{ Señales $\mathbf{( c m}^{-1} \mathbf{)}$} \\
\cline { 2 - 4 } \multicolumn{1}{c}{ Asignaciones y observaciones } & $\begin{array}{c}\text { Muestra } \\
\text { antes de } \\
\text { reticular }\end{array}$ & $\begin{array}{c}\text { Muestra } \\
\text { estabilizada }\end{array}$ & $\begin{array}{c}\text { Muestra estab. } \\
\text { después de la } \\
\text { biosorción de Pb }\end{array}$ \\
\hline $\begin{array}{l}\text { Banda correspondiente al grupo } \\
\text { funcional OH }\end{array}$ & 3305,99 & 3305,99 & 3305,99 \\
$\begin{array}{l}\text { Banda correspondiente al enlace C-H } \\
\text { alifático. }\end{array}$ & 2918,30 & 2924,09 & 2933,77 \\
$\begin{array}{l}\text { Banda correspondiente al estiramiento } \\
\text { asimétrico del grupo -COO. }\end{array}$ & 1728,22 & 1600,92 & 1573,91 \\
$\begin{array}{l}\text { Banda correspondiente al estiramiento } \\
\text { simétrico del grupo -COO. }\end{array}$ & - & 1415,75 & 1409,96 \\
$\begin{array}{l}\text { Banda correspondiente al estiramiento } \\
\text { del enlace C-O. }\end{array}$ & 1031,92 & 1029,99 & 1026,13 \\
\hline
\end{tabular}

\section{Análisis SEM - EDAX}

El análisis con espectroscopía de rayos X con energía dispersiva, EDAX (Energy-dispersive $\mathrm{X}$-ray spectroscopy), permite determinar la composición elemental de los elementos existentes de un material determinado y el método de microscopía electrónica de barrido, SEM (Scanning Electron Microscopy) puede ser empleado para estudiar la morfología de las superficies. Con ambas técnicas se realizó la caracterización de la superficie del biosorbente antes de su estabilización con cloruro de calcio y después y, posteriormente, después de la biosorción de plomo.

Las figuras 3, 4 y 5 muestran las microfotografías SEM del alga Ascophyllum nodosum en 
las tres etapas del proceso. La caracterización morfológica indicada por las microfotografías nos indica la heterogeneidad de las partículas. En los espectros EDAX se observa una señal muy intensa de $\mathrm{C}$ y otra de menor intensidad de $\mathrm{O}$ características en el biopolímero. El pico del elemento $\mathrm{Pb}$ puede observarse en la figura 5; también se detectó picos de $\mathrm{Au}$ en los tres espectros debido al tratamiento con polvo de oro utilizado antes de la medición.
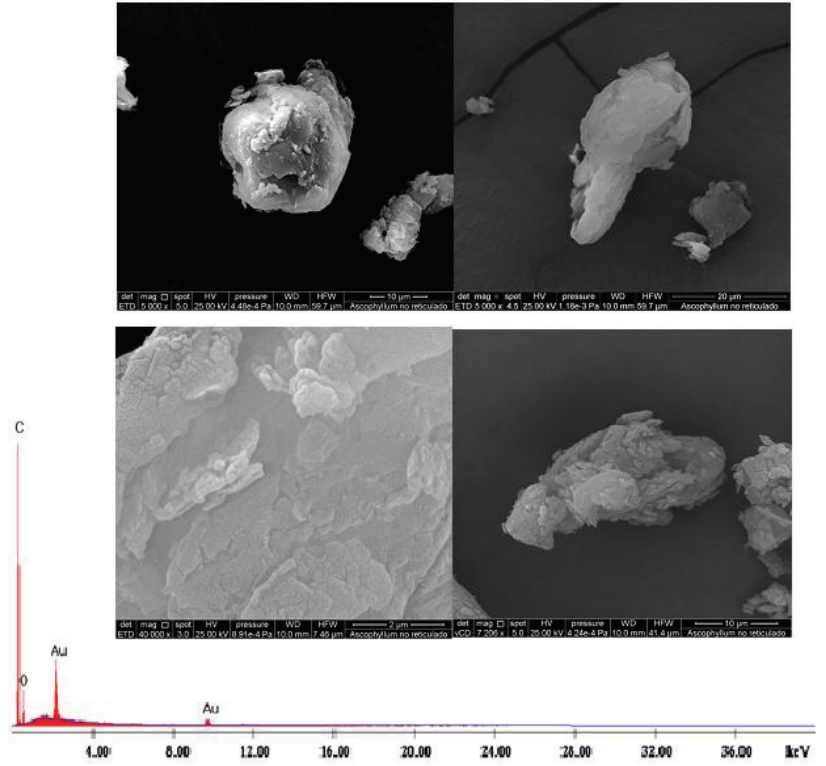

Figura 3. Microfotografía SEM y espectro EDAX del Ascophyllum nososum ckedax327genesisigenspc.spc

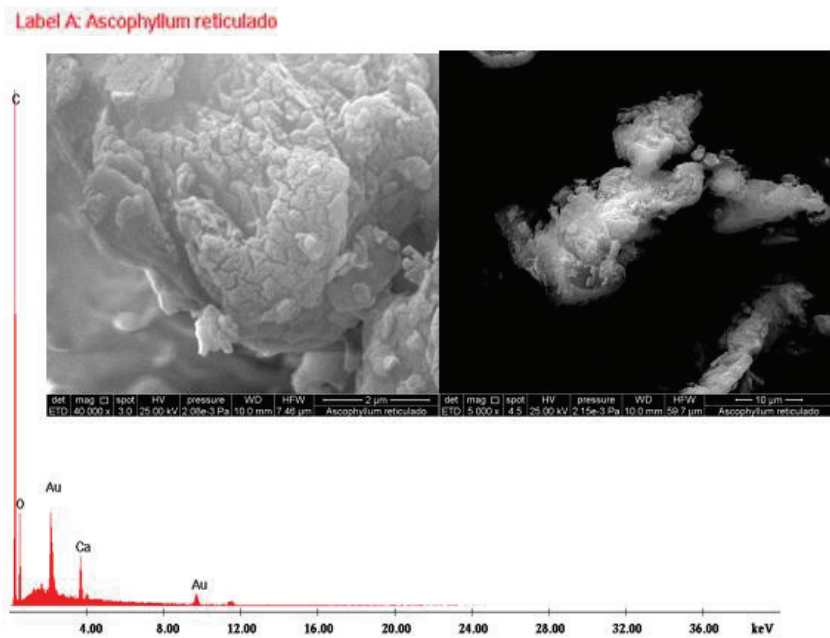

Figura 4. Microfotografía SEM y espectro EDAX del Ascophyllum nososum estabilizado 


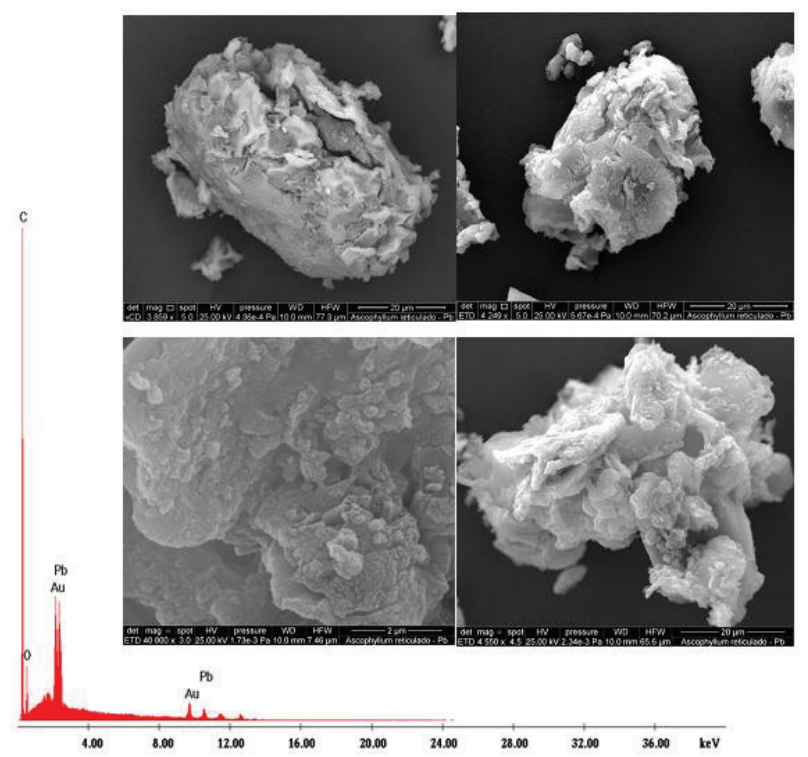

Figura 5. Microfotografía SEM y espectro EDAX del Ascophyllum nodosum estabilizado después de la biosorción del plomo
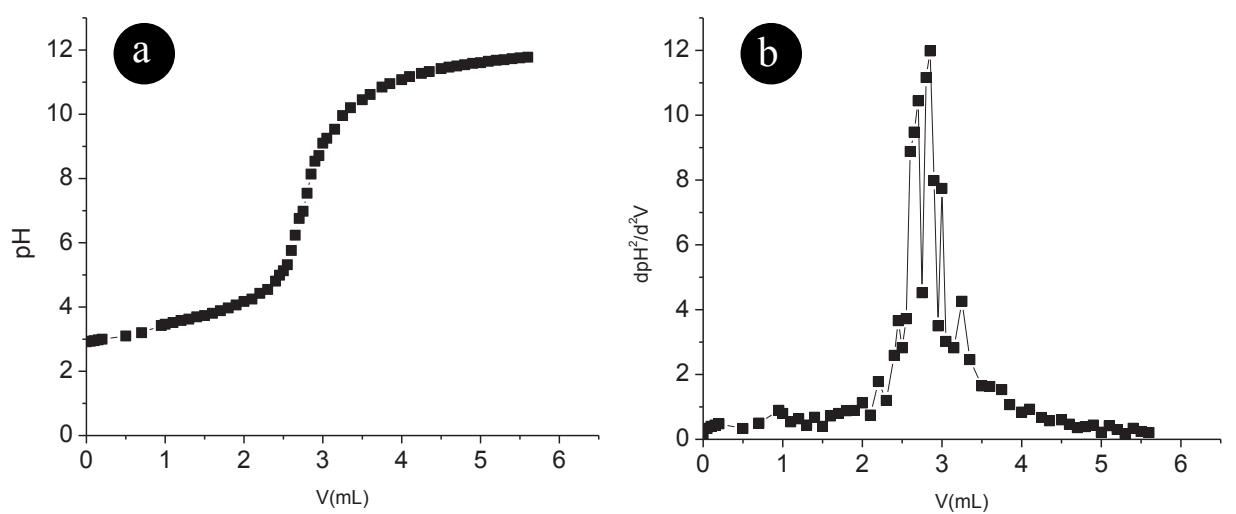

Figura 6. a. Neutralización del Ascophyllum nodosum. Volumen valorante, $V_{A}=2.85$ $\mathrm{mL}, p K a=3.71$. b. Primera derivada de a.

\section{Equilibrio ácido - base del alga Ascophyllum nodosum}

Sheng et al. ${ }^{8}$ determinaron que los grupos funcionales que se unen a metales pesados en las algas marinas son carboxilo, fosfatos y ácidos sulfónicos, bajo las formas de proteínas fosfatadas, alginato y fucoidanos cuyos $p K a$ son 3-4; 3,5-4,5 y 1-2, respectivamente. ${ }^{8,9}$ En la figura 6a se observa que el punto de equivalencia es alrededor del $\mathrm{pH} 8$, pudiendo corresponder a distintos grupos funcionales característicos en las algas marinas. El $p K a$ obtenido se encuentra dentro del intervalo de $p K_{a}$ que presentan los ácidos carboxílicos 
simples $(3,75-4,89) .{ }^{10}$ Esta propiedad sugiere que los alginatos contienen un considerable grado de disociación al igual que los ácidos carboxílicos simples, permitiendo la quelación con especies catiónicas como las que forman los metales cuando los grupos carboxilos se encuentran desprotonados.

\section{Estudio del equilibrio de biosorción}

La isoterma de adsorción de plomo en Ascophyllum nodosum está representada en la figura 7. La línea continua representa el ajuste a la ecuación de la isoterma de Langmuir y la línea punteada representa el ajusta a la ecuación de la isoterma de Freundlich. La tabla 2 resume los parámetros para ambos modelos obtenidos a partir del procesamiento de datos empleando el programa estadístico Origin 6.0. El coeficiente de correlación no lineal del ajuste al modelo de Langmuir es mayor al que se obtiene cuando se hace el ajuste al modelo de Freundlich. Este valor confirma la alta afinidad que presentan las algas pardas por el $\mathrm{Pb}$ (II) en la monocapa. La capacidad máxima de sorción obtenida en este estudio $(\mathrm{q}=227,56 \mathrm{mg} / \mathrm{g})$ para el $A$. nodosum excede en un $29 \%$ a la capacidad máxima obtenida por Romera et al.11 al emplear mayores concentraciones de metal y biosorbente a las mismas condiciones de $\mathrm{pH}$ y temperatura. De igual forma, los valores de capacidad máxima de sorción son ligeramente mayores a los hallados para Fucus vesiculosus descritos por Mata ${ }^{11}$.

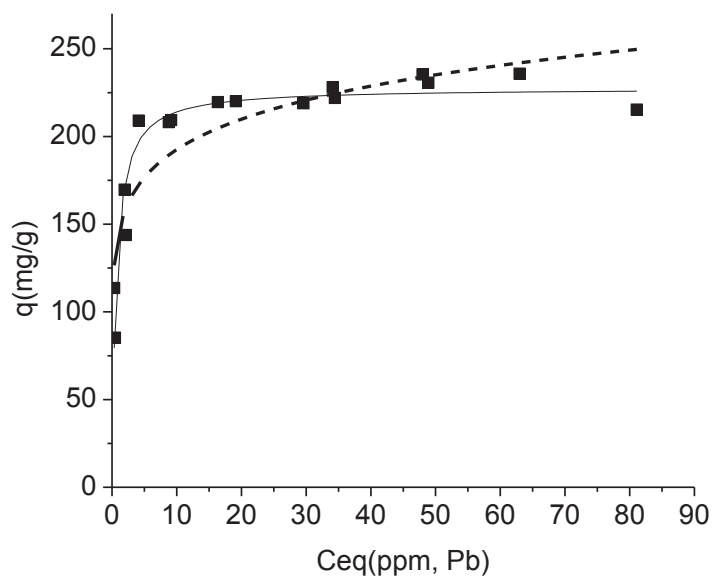

Figura 7. Isoterma de adsorción de $\mathrm{Pb}$ (II) en Ascophyllum nodosum estabilizado. La curva de tendencia para el modelo de Langmuir está representada por la línea continua

(—) y para el modelo de Freundlich está representada por la línea punteada (- - - - ).

A diferencia de la curva ajustada al modelo de Langmuir en donde se presenta una tendencia asintótica hacia el valor de la qmax a medida que aumenta $C_{e q}$, la curva ajustada al modelo de Freundlich no presenta una región de convergencia. Por este motivo, el modelo de isoterma de Freundlich es válido únicamente para sistemas en donde aún no se ha alcanzado el equilibrio.

Aunque la constante de Langmuir es dependiente de las condiciones experimentales tales como el pH de la solución y temperatura, representa un buen indicador en la comparación 
de procesos empleando diferentes biosorbentes y un mismo sorbato metal. Otro factor importante en el análisis del biosorbente es el gradiente inicial de la isoterma de adsorción. En la ecuación de Langmuir, este gradiente inicial corresponde a la constante de afinidad, $b$. Un alto valor de esta constante de afinidad favorece el proceso.

Tabla 2. Parámetros de las ecuaciones de Langmuir y Freundlich (tamaño de partícula: $0-75 \mu \mathrm{m}, \mathrm{pH} 4)$.

\begin{tabular}{cccccccc}
\hline \multicolumn{3}{c}{ Isoterma de Langmuir } & & \multicolumn{3}{c}{ Isoterma de Freundlich } \\
\cline { 1 - 2 } \cline { 5 - 7 } $\boldsymbol{q}_{\boldsymbol{m a x}}(\mathbf{m g} / \mathbf{g})$ & $\boldsymbol{b}(\mathbf{L} / \mathbf{m g})$ & $\mathbf{R}^{\mathbf{2}}$ & & $\boldsymbol{k}^{\mathbf{a}}$ & $\boldsymbol{n}$ & $\mathbf{R}^{\mathbf{2}}$ \\
\hline 227,585 & 1,578 & 0,9074 & & 144,695 & 8,058 & 0,7950 \\
\hline
\end{tabular}

a Unidades de $\mathrm{k}: \mathrm{mg}^{1-1 / \mathrm{n}} \mathrm{g}^{-1} \mathrm{~L}^{1 / \mathrm{n}}$

\section{CONCLUSIONES}

El alga Ascophyllum nodosum tratada con cloruro de calcio resultó ser un biosorbente eficaz para la remoción de plomo (II). A pH inicial entre 4 y 5 la máxima capacidad de adsorción alcanzada fue $230 \mathrm{mg} / \mathrm{g}$.

Los resultados obtenidos en el equilibrio de biosorción presentaron un coeficiente de correlación mayor, $\mathrm{R}^{2}$, para el modelo matemático de Langmuir, verificando, tal como señala Castellan ${ }^{12}$, que la adsorción química no va más allá de una monocapa en la superficie, por lo que la isoterma de Langmuir es más apropiada para interpretar estos datos.

\section{AGRADECIMIENTO}

Los autores agradecemos al Departamento de Ciencias Exactas-Sección Química de la Facultad de Ciencias y Filosofía Alberto Cazorla Talleri de la Universidad Peruana Cayetano Heredia y al Departamento de Química Analítica de la Facultad de Química e Ingeniería Química de la Universidad Nacional Mayor de San Marcos por el apoyo brindado en la presente investigación.

\section{BIBLIOGRAFÍA}

1. Viglio, F., Beolchini, F., Removal of metals by biosorption: 2 Review. Hidrometallurgy, 1997;44: 301-306.

2. Volesky, Bohumil, Sorption and Biosorption. Published by Bv Sorbex, Inc. - MontrealSt Lambert, Quebec, Canadá, 2003.

3. Volesky, B; Holan, ZR., Biosorption of heavy metals, Biotechnol. Progr., 1995;11(3): $235-250$.

4. Pagnanelli, F., Esposito, A., Toro, L., Viglio, F., Metal Speciation and $\mathrm{pH}$ effect on $\mathrm{Pb}$, $\mathrm{Cu}, \mathrm{Zn}$, and $\mathrm{Cd}$ biosorption onto Sphaeratilus natans: Langmuir-Type empirical model. 
Water Research, 2003;37: 627-633.

5. Tapia, N., Aplicaciones ambientales de la biotecnología en la industria: Adsorción y Biosorción, Mecanismos y principales modelos para describir el fenómeno de biosorción, Proyecto Palma, PUCP, 3, 5 y 6 de agosto, 1 - 17. 2002.

6. Guibal, E., "Biosorción y adsorbentes para el tratamiento de efluentes minerometalúrgicos e industriales", Curso internacional, Universidad Peruana Cayetano Heredia, Facultad de Ciencias y Filosofía. 1997.

7. Davis, A., Volesky, B., Mucci, A., A review of the biochemistry of heavy metal biosorption by brown algae, Water Research, 2003;37: 4311-4330.

8. Pin Xin Sheng, Yen-Peng Ting, J. Paul Chen, Liang Hong., Sorption of lead, cooper, cadmium, zinc and nickel by marine algal biomass: Caracterization of biosorptive capacity and investigation of mecanisms, Journal of Colloid and Interface Science, 2004;275:131-141.

9. WHO (World Health Organization). WHO Guidelines for Drinking Water Quality. 3rd Edition. Recommendations. 2004.

10. Wade, L., Química Orgánica. Ed. Prentice Hall Hispanoamericana, S. A. 2002.

11. Romera, E., González, F., Ballester, A., Blázquez, M. L. \& Muñoz, J., Comparative study of biosorption of heavy metals using different types of algae. Bioresource Technology, 2007; 98: 3344-3353.

12. Castellan, G.W. Physical Chemistry. Massachusetts. Addison-Wesley Publishing Company. 1983. 